potentially severe consequences. Care home residents are particularly vulnerable to inappropriate prescribing. With a growing ageing population, strategies to improve prescribing are essential. The aim of this systematic review was to collect and interpret the results of controlled trials of interventions to reduce inappropriate prescribing in care homes, to determine the most effective strategies. Method Databases searched were MEDLINE, EMBASE, international pharmaceutical abstracts and the Cochrane library. Search items included "nursing home", "residential home", "inappropriate prescribing", "education", "staff education", "MDT", "pharmacist", "computer". The search strategy retrieved 16 articles that met the inclusion criteria. Two independent reviewers undertook screening and methodological quality assessment, using the Downs and Black rating scale. A meta-analysis could not be done due to heterogeneity of the outcome measures used in the different studies.

Results Four intervention strategies were indentified: education, multidisciplinary team (MDT) meetings, clinical pharmacist reviews and computerised clinical support systems. Educations interventions was the most studied area, with six studies showing an improvement in inappropriate prescribing. Mixed results were found for the pharmacist interventions, possibly due to the inappropriate choice of outcome measures used for assessing prescribing quality. Computerised decision support systems were evaluated in two studies, with one showing a significant increase in the final appropriate drug orders. Two of the three studies examining MDT meetings found an overall improvement in quality of prescribing.

Conclusion Results from various interventional strategies are mixed; a multi-faceted approach, clearer policy guidelines and standardised measurements for measuring inappropriate prescribing are required to improve prescribing practices for these vulnerable patients.

\section{P27 OLDER ADULTS WITH CANCER-ARE THOSE WHO LIVE ALONE AT THE END OF LIFE A DISADVANTAGED GROUP? A QUALITATIVE STUDY}

doi:10.1136/jech.2010.120477.27

\begin{abstract}
${ }^{1} \mathrm{~L}$ Cooper, ${ }^{2} \mathrm{~J}$ Addington-Hall, ${ }^{3} \mathrm{~A}$ Arthur, ${ }^{4} \mathrm{G}$ Grande, ${ }^{5} \mathrm{~S}$ Payne, ${ }^{3} \mathrm{~J}$ Seymour, ${ }^{1} \mathrm{~B}$ Hanratty. ${ }^{1}$ Division of Public Health, University of Liverpool, Liverpool, UK; ${ }^{2}$ School of Health Sciences, University of Southampton, Southampton, UK; ${ }^{3}$ School of Nursing, Midwifery \& Physiotherapy, University of Nottingham, Nottingham, UK; ${ }^{4}$ School of Nursing, Midwifery \& Social Work, University of Manchester, Manchester, UK; ${ }^{5}$ Division of Health Research, Lancaster University, Lancaster, UK
\end{abstract}

Health status will help to determine where an older adult can live, but it is also possible that living arrangements may themselves have consequences for the health experience of older adults towards the end of life. This study will explore the relevance to cancer patients of factors that the literature suggests might account for poor health and different patterns of service use amongst solitary living older people.

Objective To determine how older adults with cancer, living alone with advanced disease, have distinct experiences, needs and preferences for care, which merit attention.

Design In-depth qualitative interviews with 30 people, half of whom live alone. Participants were recruited from general practices and day hospices in the North West, aged over 75 years; with a documented diagnosis of cancer and professionally determined prognosis of less than 12 months. Baseline face-to-face interviews were followed by telephone contacts at 3 and 6 months. Data were analysed using Framework, a matrix-based approach.

Findings Many of the older adults in this study described substantial networks of intergenerational, spousal and neighbour support, irrespective of living arrangements. Those who lived alone had high levels of functioning and quality of life despite poor health status and practical challenges. The desire to maintain independence in all aspects of their lives was a key theme. Loneliness and social isolation were not common, with few differences observed with living arrangements. Companion animals played an important social role and helped to define the daily routine for some participants who lived alone.

Conclusion Living arrangements are easily observed, and a convenient way of defining a population for study. However, the availability of familial and community support may be a more important influence on older people's end of life experiences. Our findings suggest that the very old living alone with cancer may be a particularly resilient group of people.

\section{P28 PATTERNS OF DIETARY SUPPLEMENT USE AMONG OLDER MEN AND WOMEN: FINDINGS FROM THE HERTFORDSHIRE COHORT STUDY}

doi:10.1136/jech.2010.120477.28

${ }^{1} \mathrm{H}$ J Denison, ${ }^{1} \mathrm{~S} M \mathrm{M}$ Robinson, ${ }^{1} \mathrm{~K}$ A Jameson, ${ }^{1} \mathrm{H}$ E Syddall, ${ }^{1} \mathrm{H} \mathrm{J}$ Martin,

${ }^{1} \mathrm{E}$ M Dennison, ${ }^{1} \mathrm{C}$ Cooper, ${ }^{1,2} \mathrm{~A}$ Aihie Sayer. ${ }^{1} \mathrm{MRC}$ Epidemiology Resource Centre, University of Southampton, Southampton, UK; ${ }^{2}$ Academic Geriatric Medicine, University of Southampton, Southampton, UK

Setting Use of dietary supplements has increased over recent decades in the UK. Although dietary supplement use has been described in a number of populations we know little about patterns of supplement use in older people.

Objective The objectives of this study were to describe dietary supplement use in the Hertfordshire Cohort Study (HCS), to determine patterns of supplement use, and to investigate the extent to which supplement user groups differ in terms of their characteristics, diets and morbidity.

Methods 3217 HCS participants, aged 59 to 73, were interviewed. Diet over the preceding 3 months was assessed by FFO; compliance with "healthy" eating recommendations was defined using individual scores for a "prudent" dietary pattern, identified using principal components analysis. Details of the brand and dose of all dietary supplements taken in the preceding 3 months were recorded. Individual supplements were allocated to one of 10 groups based on their nutrient composition, and cluster analysis was used to define groups of supplement users. The demographic and lifestyle characteristics, and morbidity of the supplement user groups was examined.

Results $45.4 \%$ of men and $57.5 \%$ of women reported taking at least one dietary supplement in the previous 3-month period; the most commonly taken type of supplement was oils (43\% of total supplement products taken), particularly fish liver oil. There were five distinct clusters of supplement users; these were common to men and women. They were labelled according to the principal supplement taken; oils, glucosamine, single vitamins, vitamins and minerals, and herbal products. For men, there were differences between the groups for BMI $(p=0.030)$, "prudent" diet score $(p=0.002)$, Hospital Anxiety and Depression score $(p=0.013)$ and social class $(p=0.012)$. For women, there were differences between the groups for age $(p=0.030)$, "prudent" diet score $(p=0.014)$ and social class $(p=0.005)$. There was no significant difference between the groups for Hospital Anxiety and Depression score among the women. With the exception of a difference in diagnosis of diabetes among the women $(p=0.021)$, there were no differences in morbidity between the supplement groups in either men or women.

Conclusion There were distinct patterns of supplement use in the HCS. Supplement user groups differed in their characteristics, but there were few differences in morbidity. 